\title{
JSAIS: AN OPEN JOURNAL
}

\author{
Craig Van Slyke \\ Northern Arizona University \\ craig.vanslyke@nau.edu
}

\author{
Michael Cuellar \\ North Carolina Central University \\ mcuellar@nccu.edu
}

\author{
Meg C. Murray \\ Kennesaw State University \\ mcmurray@kennesaw.edu
}

Launching a new journal is an intimidating prospect, especially in an increasingly-crowded journal marketplace. To be successful (more on "success" later), a new journal must establish an identity; it must stand out from the crowd in some meaningful, positive way. In this editorial, we discuss the manner in which the Journal of the Southern Association for Information Systems (JSAIS) seeks to stand out and contribute to the field of information systems.

\section{MISSION AND IDENTITY}

The Journal of the Southern Association for Information Systems (JSAIS) is sponsored by the Southern Association for Information Systems (SAIS), the first and oldest AIS chapter. JSAIS will emphasize originality, importance, and cogency of ideas, with a broad focus emphasizing various research methodologies and inclusive of interdisciplinary investigations. JSAIS sets to distinguish itself by employing an open and innovative review process designed to be inclusionary. JSAIS is committed to working closely with authors to develop submitted work while adhering to the high scholarly research standards.

\section{OPEN}

If we could only use one word to describe the philosophy behind JSAIS it would be "open." "Open" is an interesting word, especially as it relates to intellectual pursuits. The Free Dictionary (http://www.thefreedictionary.com/open) includes several definitions of open that are particularly interesting in our context:

- Accessible to all

- Available for use

- Carried on in full view

- Free from limitations, boundaries or restrictions

- Willing to consider or deal with something

These definitions for "open" provide a framework for discussing how JSAIS seeks to differ from existing outlets and contribute to the field.

\section{Access}

Two of our definitions pertain to access, accessible to all and available for use. JSAIS will be freely available online. We will not charge a subscription fee. While we hope many readers will be encouraged to join the Southern Association for Information Systems (JSAIS' sponsoring organization), we believe that it is important to keep the journal subscription free. There are two reasons for this. First, we believe that it is an ethical matter to provide access to all, regardless of their financial resources. The second reason is more pragmatic. By making the journal freely available, we hope to increase the influence of the journal; easier access should lead to more readers. 
"Unless one is a genius, it is best to be intelligible." - Sir Anthony Hope Hopkins

The second relevant aspect of access pertains to the tone of JSAIS articles. Scholarly articles are sometimes criticized for being difficult for non-academics to read and understand. Sometimes this is unavoidable due to the prerequisite knowledge needed to grasp the underlying meaning of an article. However, we believe that many academic works are unnecessarily dense with respect to the writing style. Unfortunately, a scholarly writing style is often equated to passive voiced, third-person prose. In our opinion, this is an outdated, unnecessary notion. Turgid prose does not equate to great content. Accessible writing is clear, concise and to the point. This is the goal we pursue at JSAIS.

Making articles more accessible (in both senses) leads to greater impact on our field, particularly with respect to practitioners. Making articles freely available and easier to read should increase JSAIS' contribution to information systems.

\section{Reviewing}

The definition "carried on in full view" also pertains to JSAIS. We employ open reviewing. Some view double-blind reviewing as the gold standard of peer review. While this sort of reviewing has its benefits, we believe that the benefits of open reviewing outweigh those of double-blind reviewing. Open reviewing provides greater transparency and accountability. It also reduces the possibility of reviewer abuse and cronyism. Of $\mathrm{c}$.ourse, there are criticisms of open reviewing as well. Our main concern with this model is that some administrators and promotion and tenure committees may hold rigid notions of "peer review." Rest assured that peer review is a cornerstone of JSAIS, but implemented using a different model of peer review.

When an author submits an article to JSAIS, we post it to a reviewing website. Once the article is posted, it is immediately available to all. We then assign at least two designated reviewers. These reviewers perform a traditional review of the entire paper. When complete, their reviews are posted with attribution. During the review period, others have the opportunity to comment on the paper. In some cases, these comments are similar to traditional reviews, but others may focus on a particular aspect of the paper. This model ensures the same quality control of traditional reviewing, but has the added benefit of "many eyes." (Given enough eyes, all bugs are shallow (Raymond, 1999).) Commenters may find ways to improve the paper beyond those suggested by the designated reviewer. Like the reviewers, the identity of commenters is known to the entire community.

\section{Free From boundaries}

The last two of our definitions of "open", free from limitations, boundaries or restrictions, and willing to consider or deal with something, relate to JSAIS' willingness to consider novel ideas. JSAIS does not favor any single research area, theory base, or methodology. Some journals have stated or unstated preferences for certain types of articles. This is not the case with JSAIS. We want interesting articles, regardless of the area or methodology used. Of course, JSAIS articles must address information systems in some way, but we take a broad view of the information systems domain. We leave debates such as the "IT artifact" argument to other journals.

\section{ADDRESSING CRITICISMS OF EXISTING JOURNALS}

Launching a new journal is a unique opportunity to address concerns about the current system. Unfortunately, there are well known weaknesses in our current system of scholarly publishing. These weaknesses have been widely discussed, especially by recent Editors-in-Chief of MIS Quarterly, including Rick Watson, Carol Saunders and Detmar Straub. In this section, we discuss some of these weaknesses and how JSAIS addresses them. (It is worth noting that many of the ideas behind JSAIS were developed during conversations with Don Hardaway, who goes into more depth on shortcomings of our current peer review process in a forthcoming MIS Quarterly article (Hardaway \& Scamell, 2012).) 


\section{Type I and Type II Errors}

Straub (2008) points out two types of problems that occur in the reviewing and publishing system. Type I errors occur when a paper is published, but not valued by the community and does not influence future knowledge creation. In contrast, Type II errors occur when a paper that would be valued by the community is rejected. As Straub mentioned, Type II errors are especially problematic because these "good" papers may never be published.

The Type I/Type II framework is interesting with respect to the costs of each type of error. The main costs of a Type I error is the noise created by uninteresting articles, and potential reputation-reducing effects on the journal. Of course, the uninteresting article may also take a publication slot that could be put to better use. Type II errors are more costly in the long run. Unpublished papers do not contribute to the field's collective knowledge.

\section{Gatekeepers or Diamond Cutters}

Traditionally reviewers and editors view their roles as gatekeepers, insuring that "bad" papers do not become part of a field's knowledge base. As Saunders (2005) discusses, this proclivity leads to a bias towards finding reasons to reject a paper, looking for the "fatal flaw" that kills the chance of publication. Saunders proposes a different role using the metaphor of a diamond cutter. The field may be better off if reviewers and editors view themselves as the experts who can find the gem in a cluster of carbon. Such developmental reviewing shifts the mindset from finding reasons to reject to finding ways to make the paper publishable. The diamond cutter perspective may reduce Type II errors.

JSAIS therefore will seek to be "developmental." The goal of the review process is to improve the paper to make it the best that it can be rather than filter out papers that "do not meet the standard for publication." Reviewers of JSAIS therefore are encouraged to not only point out defects in the paper but also to suggest how this defect can be remedied. The reviewer as diamond cutter seeks to remove the dross in the form content that doesn't belong in the paper, incorrect interpretation or omission of literature, or bad methodology. He or she also seeks to polish the stone by assisting in improving the writing in style, structure and construction. While the reviewers are not an editor or grammarian, they are expected to provide assistance on how to revise the manuscript to be more accessible in terms of readability. Finally, reviewers are free to cut the diamond by perhaps suggesting that a paper be divided into two or more papers that are more coherent. Each of which will be more valuable than the original.

\section{Novelty vs. Rigor}

Kohli and Straub (2011) offer several "sparkles emanating from the diamonds in the rough." These include novel ideas, innovative methods, interesting contexts and informing IS practice. Essentially these boil down to papers that offer something new or novel, or that impact practice.

Therefore, we seek to publish "interesting" papers. Davis (1971) suggests several ways in which a paper is interesting. Essentially, an interesting paper is something that rises above the clutter of the humdrum and mundane to show us something new or novel especially when it negates the "received" or "common sense" view and propounds a different way of viewing reality. Davis suggests such novelties may be found in several ways. For example, showing that what seems to be organized in a certain way is really organized in a different way or not organized at all. Or what seems to be all one thing is actually several things or vice versa. What is taken to be a "good" phenomenon is actually a "bad" one and vice versa. Things that seem to be unrelated are actually related, etc. JSAIS is especially looking for papers like these. We are open to the paradigm shift, the unconventional thought, and the iconoclastic vision. We are not so much interested in papers that add another variable to the TAM model or validate the Delone and McLean IS success model as we are in papers that offer alternatives to those models that may explain the phenomena better. Even if not fully fleshed out, let those ideas come here to be developed.

Traditionally, few journals allow "novelty" to trump rigor. Many IS research outlets focus too much on papers that are technically sound, but offer little impact on the broader world. In our experience, many 
(perhaps most) IS journals seem to favor technical rigor or conformance to established paradigms over the novelty of ideas. (Of course there are notable exceptions to this.) We believe there is a need for a new journal that favors interesting ideas, even if they are not fully developed. This is not to say that JSAIS will ignore rigor, rather we will be willing to publish papers that offer interesting ideas, even if there are methodological weaknesses. As long as authors acknowledge the weaknesses and consider the implications of the weaknesses, JSAIS will still consider publication. We will expect authors to fix methodological problems to the extent reasonably possible.

\section{Community Reviewing}

The community reviewing aspect of JSAIS also helps address the issue of finding diamonds in the rough. Even if a journal adopts the reviewing mindset proposed by Saunders and Straub, only a small number of reviewers/editors help refine the paper. By adopting a community reviewing model, we seek to take advantage of the "many-eyes" concept from the open source software community (Raymond, 1999). This concept states that with many eyes, all bugs are shallow. Allowing community input ideally takes advantage of "many-eyes" in two important ways. First, community members may see value that the formal reviewing team missed. Second, community members may identify solutions to a paper's weaknesses. Overall, this approach potentially leads to stronger articles (Hardaway, 2005).

Another interesting aspect of community reviewing is that a community reviewer may choose to focus on a narrow aspect of a paper. Formal reviewers must consider the paper in its entirety. A community reviewer is not restricted in the same way. As a result, a community member who has deep expertise or interest in one part of a paper may restrict her/his comments to that aspect. This is important, especially for a new journal. It is difficult to find reviewers who have deep expertise in all aspects of a paper. By allowing community members to comment on a paper during the reviewing process, the authors have the benefit of tapping a broad array of expertise.

It is worth noting that there are critics of the many-eyes concept. Animal behavioralists proposed a manyeyes hypothesis that basically states that as group size grows, individual animals are less vigilant about detecting the presence of predators. Individual group members take less responsibility for detecting unfavorable conditions, possibly believing that others will assume the responsibility. Human behavior exhibits some of the same characteristics as proposed, in concepts such as diffusion of responsibility and the bystander effect. Glass (2002) carries this into the software engineering realm. He contends that research shows that more code inspectors actually lead to fewer errors being detected. Having a small number of formal reviewers is a more effective strategy.

Both of these positions have merit. In the case of academic reviewing, it does not have to be an either-or proposition. At JSAIS we have both formal, assigned reviewers and more informal community reviewers (which we call commenters). Our hope is that allowing both roles takes advantage of both perspectives. Formal reviewers are specifically tasked with carrying out the deep review, while community members are free to more casually comment on portions of the paper. (Of course, some community members may carry out more extensive reviews.)

\section{CRITICAL SUCCESS FACTORS}

The level of success JSAIS achieves depends on many factors. In this section, we briefly discuss several of these critical success factors.

\section{Submissions}

Quality submissions are the foundations of a quality journal. To be successful, JSAIS must receive a critical mass of submissions. Achieving critical mass is especially challenging for a new journal. JSAIS is not on journal lists, not listed in Cabells nor does it have an impact factor. Unfortunately, these facts may make JSAIS a less attractive choice for some authors. This is a bit of a "chicken-and-egg" situation. We need good articles to build our reputation, but reputation is what attracts high-quality articles. 
We encourage those of us with more flexibility in choosing outlets to submit their works to JSAIS. Full professors and faculty at schools with more generous publishing criteria can help JSAIS build its reputation by submitting their articles. Over time, good articles attract good submissions, enhancing JSAIS' reputation in the community.

The developmental nature of JSAIS makes it an attractive outlet for newer researchers, such as graduate students. Fortunately, the fresh eyes of newer members of our community often lead to interesting, novel perspectives. As noted earlier, articles that present such views are particularly attractive to JSAIS.

Faculty who are reviving their research should also consider submitting to JSAIS. For example, administrative assignments often leave little time for research. When these assignments end, faculty may need to retool with respect to research. Our developmental review and editing process may be helpful in these situations.

\section{Participation}

Participation by community members is also critical to our success. In addition to submitting articles, JSAIS also needs active reviewers and commenters. Attracting committed reviewers is a challenge for many journals. Reviewing is a time consuming, challenging task for which there is little extrinsic reward, but quality reviews are prerequisite to a quality journal. We wish we could offer more than intrinsic satisfaction to our reviewers, but we simply cannot. We simply appeal to the better nature of our community reviewers and ask them to "pay it forward" by providing thoughtful, timely reviews.

Sometimes, however, people do not have the time, interest or knowledge to provide a full review of an article. However, these individuals may be able to comment on specific aspects of the paper. The commenting feature of our review process lets community members contribute in a smaller, but still meaningful way.

\section{Readers}

A journal without readers is largely pointless. So, JSAIS needs readers. We encourage you to periodically browse JSAIS to find articles of interest. Sharing article with your colleagues is also helpful. Social networking tools provide easy means to share. Mentioning a JSAIS article in a twitter feed, sharing it with a LinkedIn group or Google+ circle helps JSAIS gain readers. We welcome other suggestion for boosting our readership.

\section{Citations}

Citations are an often-used measure of a journal's influence. As a result, we encourage you to reference JSAIS articles when appropriate. The words "when appropriate" are critical. JSAIS will NOT require authors to cite JSAIS, nor will we consider JSAIS citations as a factor when making a publication decision. Of course, we do appreciate citations of JSAIS articles, but only when appropriate.

\section{CONCLUSION}

Your editorial team is excited to launch our first issue. We appreciate the assistance of those who have helped us get this far. To those who will help in the future, we offer our "thanks in advance." For JASIS, becoming a publication of choice is our goal. We hope to build JSAIS into an outlet known for publishing interesting, timely, well-constructed articles. Achieving this goal requires the community's help. We hope that you will help us on the upcoming journey.

\section{REFERENCES}

1. Davis, M.S. (1971) That's interesting. Philosophy of Social Science, 1, 309-344. http://dx.doi.org/10.1177/004839317100100211

2. Glass, R.L. (2002) Facts and Fallacies of Software Engineering, Addison-Wesley, Boston, MA. 
3. Hardaway, D. (2005) Sharing Research in the 21st Century: Borrowing a Page from Open Source Software, Communications of the ACM, 48, 8, 125-128. http://dx.doi.org/10.1145/1076211.1076216

4. Hardaway, D. and Scamell, R. (2012) Open knowledge creation: Bringing transparency and inclusiveness to the peer review process, MIS Quarterly, 36, 2, 339-346.

5. Kohli, R. and Straub, D. (2011) Editor's comments: How reviews shape MIS Quarterly: A primer for reviewers and editors, MIS Quarterly, 35, 3, iii-vii.

6. Raymond, E.S. (1999) The Cathedral \& the Bazaar: Musings on Linux and open source by an accidental revolutionary, O’Reilly Media, Sebastopol, CA.

7. Saunders, C. (2005) Editor's comments: Looking for diamond cutters, MIS Quarterly, 29,1, iii-viii.

8. Straub, D. W. Editor's comments: Type II reviewing errors and the search for exciting papers, MIS Quarterly, 32, 2, v-vii. 